\title{
Should Allogeneic Hematopoietic Stem Cell Transplantation be a Treatment Option for Patients with Nijmegen Breakage Syndrome? Belarusian Experience
}

\author{
Olga V. Aleinikova, Alina S. Fedorova, Svetlana O. Sharapova \\ Belarusian Research Center for Pediatric Oncology, Hematology and Immunology, Minsk Region, Belarus
}

Olga V. Aleinikova, MD, PhD, Director of the Belarusian Research Center for Pediatric Oncology, Hematology and Immunology, 43, Frunzenskaya Str., Borovlyany, Minsk region, 223053, Belarus.
Tel.: 00375-17-2654222

Fax: 00375-17-2654222

e-mail: aleinikova2004@mail.ru

\section{Summary}

Introduction: Nijmegen breakage syndrome (NBS) is characterized by chromosome instability, combined immunodeficiency, radiosensitivity and high predisposition to lymphoid malignancy. A specific therapy is not available, however, hematopoietic stem cell transplantation (HSCT) is considered for NBS patients with refractory or recurrent leukemia or lymphoma.

Purpose: We aimed to present Belarusian data in NBS diagnosis and management and to discuss indications for allo-HSCT treatment.

Patients and Methods: A total of 238 patients were registered with primary immune deficiency (PID), including 19 cases of NBS. DNA was analyzed for mutation in NBN gene by direct sequencing of exon 6 . Histological classification of lymphoid neoplasms was performed according to World Health Organization classification (2008). The patients were treated according to modified pediatric regimens or individually.

Results: NBS accounted for $8.0 \%$ of all PID cases and was diagnosed at the age from 0.3 to 21.6 years (median 7.1). Mutation 657-661delACAAA in NBN gene and combined immunodeficiency of various degrees was confirmed in all patients. Lymphoid malignancy developed in 9 (47.4\%) NBS patients ageing from 4.3 to 21.6 years (median 10.7). Acute leukemia was diagnosed in 4 patients, and stage III
non-Hodgkin's lymphoma in 5. Lymphoma/leukemia were in 7 out of 9 cases of T-cell origin. Complete remission (CR) was achieved in $66.7 \%$ of the patients.. Events were: death from infections in induction or in CR1 in 3 patients, progression/relapse in 3 , second lymphoma in 1 . Totally 7 NBS patients died, all after the development of malignancies. Two patients with T-mature lymphoma/leukemia did not respond to induction chemotherapy, were treated with unrelated HSCT in the 1CT and alive disease free within 6 and 13 months after transplantation. HSCT from a sibling donor was performed 9.8 years ago in a boy without malignancy but with infectious and autoimmune complications. They all received reduced-intensity conditioning regimens, which were well tolerated.

Conclusions: NBS needs to be diagnosed early. With respect to progressive immunodeficiency and high risk of lymphoid malignancies with uncertain curative prospective, an allo-HSCT approach with reduced-intensity conditioning could be proposed as a treatment option for NBS patients with severe defects of immune function, and for all NBS patients with lymphoid malignancy in $1^{\text {st }}$ complete remission.

\section{Keywords}

Nijmegen breakage syndrome, immunodeficiency, lymphoid malignancy, stem cell transplantation

Running title: Stem cell transplantation in the Nijmegen syndrome. 


\section{Introduction}

Nijmegen breakage syndrome (NBS) is a rare autosomal recessive chromosome instability disorder which occurs worldwide with prevalence among Eastern and Central European population and caused by mutation in $N B N$ gene at 8q21, the most often mutation 657-661delACAAA in 6 exon, named Slavic mutation [1-4]. Except of progressing microcephaly, low birth weight, growth retardation and characteristic bird-like facial features, it is characterized by combined immunodeficiency, radiosensitivity and high predisposition to malignancy development in childhood, mostly of lymphoid origin [5-6]. NBN gene encoding the nibrin protein plays a key role in DNA double-strand break repair and the maintenance of genomic stability. Insufficient function of defective nibrin decreases cellular response to DNA damage and results in genomic instability and finally can lead to cancer, which remains the most common cause of death in patients with NBS [7-8]. Other known causes of death are fatal infections and bone marrow aplasia [8]. Currently, no specific therapy is available for NBS. Substitution therapy with immunoglobulins is indicated in patients with agammaglobulinemia. Antibiotic prophylaxis is considered in patients with recurrent respiratory tract and urinary tract infections. Cancer treatment is accompanied by severe toxic complications and should be adapted to individual risk factors and tolerance [9-13]. The NBS patients are usually not subject to hematopoietic stem cells transplantation (HSCT), owing to concerns about increased toxicity. European experience of HSCT performed with reduced-intensity conditioning regimens in order to correct severe immunodeficiency or to treat refractory or recurring malignancy is limited but quite successful [14-15]. But there is still no consensus on optimal management of NBS patient, and no recommended options about benefits of HSCT and appropriate protocols for individual patients.

The aim of our review article was to share our experience in NBS management and to discuss indications for allo-HSCT performance. We presented here general clinical characteristics, laboratory data, treatment details and clinical outcomes of 19 Belarusian NBS patients.

\section{Patients and Methods}

According to data, provided by the Belarusian National Children Registry of primary immune deficiency (PID) diseases, a total of 238 children were diagnosed with PID until December 2014, including 18 cases of NBS. A single adult patient (21 years old) was diagnosed in our center as having NBS. The diagnosis of PID was based on the diagnostic PID criteria, as described in the last classification issued by the International Union of Immunological Societies Expert Committee for Primary Immunodeficiencies, 2013 [16].

To reveal and classify the PID condition, we used immunological criteria as well as genetic studies. Serum concentrations of immunoglobulins IgG, IgA and IgM were measured by immunonephelometry. Analysis of peripheral blood lymphocyte subpopulations was performed by flow cytometry.
The blood cells were incubated with specific monoclonal antibodies (MoAbs) against CD45, CD3, CD4, CD8, CD16/ CD56, CD19, CD31, CD45RA (obtained from Beckman Coulter and Beckton Dickinson). Recent thymic emigrants (RTE) were determined as CD4+CD45RA+CD31+ T cells.

Quantification of TRECs and KRECs was determined by RT-qPCR. Healthy age-matched donors of peripheral blood lymphocytes $(n=4)$ were taken as a control. RT-qPCR data were normalized with albumin control gene and calculated by $2^{-\Delta C t}$ [17]. Average values of TREC/KREC expression in healthy control group were taken as $100 \%$, the patients' results were presented as a percentage of control values. Statistical analysis was performed by Mann-Whitney $U$ test and Spearman rank test.

Mutational analysis of exon 6 of the NBN gene was performed in all patients. Chromosomal instability assays reflecting increased chromosomal breakage in peripheral lymphocytes was evaluated in 5 patients. Chromosomal somatic mutations (translocations and/or inversions involving chromosomes 7 and 14) were revealed in 6 cases by conventional G-banding.

Nine NBS patients were diagnosed as having acute leukemia or non-Hodgkin lymphoma (NHL). Histological classification of haematopoietic neoplasms was performed and revised, according to World Health Organization classification, 2008 [18]. Immunophenotyping was carried out in eight cases by immunohistochemical analysis or by flow cytometry. The NHL staging was based on St.Jude criteria [19]. Chemotherapy was performed according to ALL-BFM 90/2000, ALL-MB 2008 (Moscow-Berlin), AML-MM 2003 (Moscow-Minsk) and NHL-BFM 90/95 regimens with some modifications [2026]. One patient with peripheral T-cell lymphoma received individual chemotherapy (see below). Clinical data and outcomes are presented as by January l, 2015.

Parents of the patients provided written informed consent for leukemia/lymphoma treatment, as well as for blood sampling and processing for PID diagnostics, in accordance with protocols approved by the local Ethical Committee.

\section{Results}

NBS accounted for $8.0 \%$ of all the registered PID cases. A specific 657del5 mutation in the NBN gene was found in all 19 patients. Patient characteristics and immunological data are presented in Table 1. NBS occurrence was nearly equal in boys and girls (M:F ratio of 1.1:1). Median age at the NBS diagnosis was 7.1 years. NBS has been revealed only in 4 patients $(21 \%)$ under 1 year old. In most cases, examination for PID and NBS was performed after recurrent infections or development of autoimmune disease, or in connection with lymphoma or leukemia diagnosis. In four cases the diagnosis of NBS was confirmed only post mortem.

Immunological investigations revealed highly variable immunological features of the NBS patients, without evident correlations between immune markers and degree of clinical immunodeficiency, or malignancy development. Abnor- 
malities in immunoglobulin concentrations were found in $62.5 \%$ of examined patients. Just one child with hypogammaglobulinemia who received regular substitution therapy with intravenous immunoglobulin had no severe infections in anamnesis. All other patients had frequent or chronic respiratory infections. All NBS patients with autoimmune pathology, and half of the children with lymphoid malignancies had decreased serum immunoglobulin level. Moreover, two patients from our cohort developed lymphoid neoplasia at the age of 16 and 21 years, despite normal immunoglobulin serum levels and absence of frequent infections in anamnesis. Two other patients (without evidence of malignancy at the age of 3.7 and 5.7 years) had low numbers of CD3+ $\mathrm{T}$ cells $(<300$ cells $/ \mu \mathrm{L})$, thus indicative for pronounced $\mathrm{ab}$ normalities of T-cell immunity, similar to severe combined immunodeficiency. Expression of TREC and KREC mRNAs was detected at very low levels in all examined patients, including 5 children without malignancies. Immunophenotyping was performed to 13 NBS patients. A reduction of T- and/or B-cell immunity was revealed in all cases but the majority of patients had normal percentage and absolute number of CD3+ T-lymphocytes. Seven patients with low RTE level were analyzed for TRECs quantity. We found statistically significant decrease of the number of TRECs in NBS patients compared with healthy donors $(p=0.01)$. And a high correlation between RTE (\%) and TRECs' level was revealed $(\mathrm{R}=0.89, \mathrm{p}<0.05)$.

Lymphoproliferative disorders occurred in $47.4 \%$ of all registered NBS patients and in $60.0 \%$ patients older than 4 years. The median age at the time of malignancy diagnosis was 10.7 years (ranged from 4 to 21). In 7 of 9 cases, NBS was diagnosed simultaneously or after the lymphoid neoplasm was confirmed. T cell malignancies prevailed in our series ( 7 out of 8 cases with identified immunophenotype, including one case with mixed phenotype, i.e., early T/myeloid leukemia), being T-mature in half of the cases. Some details of treatment and outcomes are presented in Table 2.

Despite full chemotherapy dosage, a complete remission (CR) was achieved only in $66.7 \%$ cases (6 out of 9 cases). Two patients died of infectious complications before CR was achieved, and one patient was lost with sepsis in the $1^{\mathrm{s}} \mathrm{CR}$, while on maintenance therapy. A patient with peripheral $\mathrm{T}$ cell lymphoma developed rapid tumor growth after shortterm partial clinical response to chemotherapy and died of progression. One girl developed a secondary lymphoma 4.5 years after the treatment of diffuse large B-cell lymphoma. Three patients (two cases of T-mature lymphoblastic lymphoma, and one patient with ETP-ALL) did not respond to standard induction chemotherapy; their blast cells also had poor in vitro sensitivity to most cytostatics, as measured by MTT-test. In these patients, CR was achieved only after intensive chemotherapy (HR-blocks). The patient with ETPALL developed early relapse prior HSCT, and two others underwent unrelated HSCT and are alive with no evidence of disease at, resp., 6 and 13 months after transplantation. Overall, only two NBS patients (22.2\%) with malignancy are alive in CR1 with a follow-up of 12 and 20 months.

All NBS patients exhibited grade 3 to 4 complications after chemotherapy. Of those, most common were neutropenic infections and hemostatic disorders. Three fatal outcomes were related to first-line treatment, two from sepsis and one patient with invasive aspergillosis and massive pulmonary bleeding. Unrecognized NBS was the main reason for non-reduced dose regimens during induction therapy.

Three patients from this cohort underwent allo-HSCT (HLA-matched sibling donor in one case, and matched unrelated donors in two cases). A graft from sibling donor was transplanted 9.8 years ago to a boy without malignancy, but with infectious and autoimmune disorders (Table 1, patient \#4). His sister with NBS died from leukemia ten years earlier. Two other patients developed T-mature ALL/NHL without response to induction chemotherapy, as mentioned above (Table 2, patients \#15,16). They all received reduced-intensity conditioning regimens (Bu $8 \mathrm{mg} / \mathrm{kg}$, FLU $150 \mathrm{mg} / \mathrm{m}^{2}$, ATG $90 \mathrm{mg} / \mathrm{kg}$; MEL 140 mg/m², FLU 125 mg/m², ATG 60 mg/kg; MEL $140 \mathrm{mg} / \mathrm{m}^{2}$, FLU $150 \mathrm{mg} / \mathrm{m}^{2}$, ATG $60 \mathrm{mg} / \mathrm{kg}$ ) that were well tolerated. Every patient experienced acute graft-versus host disease (GvHD) grade II-III. Extensive chronic GvHD developed in 2 patients. Cytomegalovirus infection occurred in 2 patients and adenovirus infection was found in one case.

Currently there are 9 NBS patients under our observation, ranging from 1.1 to 20.7 years of age, who did not undergo HSCT and have no signs of malignant disorders. Unfortunately, there are no data of any living older patients with NBS in Belarus.

\section{Discussion}

NBS is a rare disease, but the number of patients identified worldwide is rapidly increasing, probably because physicians are becoming more aware of the disorder. The frequency of NBN gene mutation carriers is rather high in the population of Slavic origin, being estimated as 1 case in 177 newborns [2]. The majority of Belarusian NBS patients are residents of the southwestern part of the country, likely as a result of a founder effect. Etiopathogenesis and clinical manifestations of NBS are well studied, but no specific therapy is available. Early diagnosis of NBS is crucial, in order to avoid even diagnostic irradiation, and to prevent severe and recurrent infections by appropriate management. Increasing number of newly diagnosed NBS cases and high risk of potential complications makes it necessary to develop optimal therapeutic strategies.

Only $21 \%$ of Belarusian patients were diagnosed as having NBS at the age less than 1 year. Moreover, $78 \%$ of NBS patients who developed lymphoma or leukemia were not recognized as immunodeficient before the malignancy became evident. Their immune status was, therefore, not tested, preventive measures were not complied, and they did not receive timely treatment with intravenous immunoglobulin. As a result, their initial infectious status supposed to be worse and made their management more challenging. Therefore, the most important thing to do is to diagnose NBS as early as possible.

Combined cellular and humoral immunodeficiency is an essential feature of the disease, unless the patients with NBS mutation had variable initial immune status with inevitably 
increasing immune insufficiency over time [27-28]. Unlike some other combined immunodeficiency syndromes, HSCT is not a recommended therapeutic approach for immune system restoration in NBS patients, due to the high risk of toxic complications. Meanwhile, life-long monitoring of the immune system allows for preventive measures and maintains the patients in good clinical condition for a long time. There are patients with very mild manifestations who do not suffer from frequent infections and do not need immunoglobulin supplementation. In most patients, however, a number of complications increase over time. Chronic infections leading to development of respiratory or renal failure, severe autoimmune pathology, or malignancy cause death of NBS patients in childhood or in younger adults [4, 28-31].

In the presented cohort, combined immunodeficiency was confirmed in all patients, whereas the degree of cellular and/or humoral immunodeficiency varied from very mild to severe with very low $\mathrm{CD} 3+$ cells counts or serum immunoglobulin levels, similar to manifestations of severe combined immunodeficiency. Severity of the complications also differed individually. Our adult NBS patient had no particular history of infections before ALL diagnosed at the age of 21 years, and his clinical condition was sufficiently satisfactory to perform full dose chemotherapy treatment and allo-HSCT (Table 2, patient \#15). We have also treated another NBS patient who received adequate immunoglobulin supplementation, but multiple infectious and autoimmune complications urged us to limit the intensity of chemotherapy in the first lymphoma episode and made it impossible to perform treatment of the second lymphoma (Table 2 , patient \#3). This diversity in immune and clinical status implies a personalized therapeutic approach. Obviously, the patients with decreased immunoglobulin concentration need a substitution treatment. But we suppose that HSCT should be taken into consideration both for NBS patients with severe cellular or humoral immunodeficiency prior to the development of malignancies or other serious complications.

Patients with NBS have very high risk of cancer in childhood, mostly lymphoid malignancies, but medulloblastoma, rhabdomyosarcoma and some other tumors have been described as well [11,32-34]. As reported by the International NBS Study Group and by the Polish NBS Registry, 40 to $60 \%$ of NBS patients develop NHL by the age of 20 years $[11,35-36]$. We have obtained similar data, with $47 \%$ of NBS patients who developed lymphoproliferative disorders. The survival of children with PID-associated NHL is much lower in comparison with immunocompetent patients and ranges from 35 to $58 \%$ [9-13]. There are no specific treatment protocols designed for patients with NBS and NHL. Due to the higher risk of treatment-related toxicity, there is a common tendency to modify treatment by reducing dosage of some chemotherapy agents. Complication rate during cancer treatment was high in our cohort of children, thus being consistent with other reports [9, 11-12]. Tolerability of chemotherapy was limited mostly by infectious status rather than toxic side effects. In future, modified chemotherapy schemes combined with monoclonal antibodies should be designed for the treatment of patients with NBS and NHL.

Relapses or refractory cases of NHL occur more often in NBS than in immunocompetent patients and represent a major cause of treatment failure [9, 11-12]. Moreover, the surviving NBS patients are at increased risk for developing a second malignancy, more often, lymphoma [11, 37]. As a result, only few patients survived the whole-scale cancer treatment. There are some data on successful treatment of refractory or relapsed lymphoma with allo-HSCT in NBS patients [15]. Five out of 6 patients have restored T cell immunity and are alive, with a median follow up of 2.2 years. It has been suggested that HSCT should be considered for NBS patients with recurrent or second lymphoma.

In the present group, $87.5 \%$ patients (7 out of 8 ) were suffering from $\mathrm{T}$ cell lymphoid malignancies with $\mathrm{T}$-mature immunophenotype in a half of cases, which is different from normal spectrum of ALL/NHL in immunocompetent children, since both $\mathrm{T}$ and $\mathrm{B}$ cell lymphoid neoplasms have been described, with some prevalence of B-cell lineage and a high recurrent disease rate in T cell malignancies [11-12, 37]. The outcome of our patients was poor with only 2 survivors (22.2\%) after allo-HSCT with less than 2 years follow-up term. The reasons for this poor outcome were either infectious complications or treatment failure. More than half of the patients (5 out of 9) with NBS and malignancy did not respond to standard full dose chemotherapy or developed early relapse. Taking into account a case of secondary lymphoma development as a late event in the present cohort, the prognosis for patients with NBS and malignancies seems to be poor initially. However, despite limited tolerability of chemotherapy and a high rate of infectious complications, we demonstrated the possibility of safe allo-HSCT performance using reduced-intensity conditioning regimens.

In spite of small group under observation, we may assume that NBS patients are prone to develop T-ALL/NHL subtypes with limited curative potential. These patients may benefit from allo-HSCT as a consolidation therapy, in order to prevent relapse and to partially restore immune system, seeking to reduce the risk of secondary malignancies.

\section{Conclusion}

Our data show that NBS needs to be diagnosed at the early age. Taking into account a progressive immunodeficiency with deteriorating clinical state and extremely high risk of lymphoid malignancy with uncertain curative potential, allogeneic HSCT with reduced-intensity conditioning may be a rational therapeutic option for NBS patients with severe defects of immune function and for any NBS patients with lymphoid malignancy in first remission.

\section{Conflict of interest}

All authors have no conflict of interest to declare.

\section{Acknowledgements}

Authors gratefully acknowledges Mikhail V. Belevtsev, Chief, Belarusian National Children Registry of PID Diseases who provided information of PID cases; Dr.Svetlana N. Aleshkevich, (patient examination and collection of immunolo- 
gical data), Maria Stsegantseva, (PCR analysis of mutations), and to the physicians from our Center.

\section{References}

1. Kleier S, Herrmann M, Wittwer B et al. Clinical presentation and mutation identification in the NBS1 gene in a boy with Nijmegen breakage syndrome. Clin Genet 2000, 57: 384-387.

2. Varon R, Seemanova E, Chrzanowska K et al. Clinical ascertainment of Nijmegen breakage syndrome (NBS) and prevalence of the major mutation, 657del5, in three Slav populations. Eur J Hum Genet 2000, 8: 900-902.

3. Mauer MH, Hoffmann K, Sperling K et al. High prevalence of the NBN gene mutation c.657-661del5 in Southeast Germany. J Appl Genet 2010, 51: 211-214.

4. Seemanova E, Passarge E, Beneskova D et al. Familial microcephaly with normal intelligance, immunodeficiency, and risk for lymphoreticular malignancies: a new autosomal recessive disorder. Am J Med Genet 1985, 20: 639-648.

5. De Miranda NF, Björkman A, Pan-Hammarström Q. DNA repair: the link between primary immunodeficiency and cancer. Ann NY Academy of Sciences 2011, 1246: 50-63.

6. Tran H, Nourse J, Hall S et al. Immunodeficiency-associated lymphomas. Blood Reviews, 2008, 22: 261-281.

7. Oertel S, Reiss H. Immunosurveillance, immunodeficiency and lymphoproliferations, Heidelberg: Springer, 2002.

8. Chinen J, Anmuth D, Franklin AR et al. Long-term follow-up of patients with primary immunodeficiencies. J Allergy and Clinical Immunology 2007, 120: 795-797.

9. Seidemann K, Tiemann M, Henze G et al. Therapy for Non-Hodgkin lymphoma in children with primary immunodeficiency: analysis of 19 patients from the BFM Trials. Med Pediatr Oncology 1999, 33: 536-544.

10. Sandoval C, Swift M. Treatment of lymphoid malignancies in patients with ataxia teleangiectasia. Med Pediatr Oncology 1998, 31: 491-497.

11. Dembowska-Baginska B, Perek D, Brozyna A et al. Non-Hodgkin lymphoma (NHL) in children with Nijmegen Breakage syndrome (NBS). Pediatr Blood Cancer 2009, 52: 186-190.

12. Bienemann K, Burkhardt B, Modlich S et al. Promising therapy results for lymphoid malignancies in children with chromosomal breakage syndromes (Ataxia teleangiectasia or Nijmegen-breakage syndrome): a retrospective survey. Br J Haematology 2011, 155: 468-476.

13. Sandlung JT, Hudson MM, Kennedy W et al. Pilot study of modified LMB-based therapy for children with ataxia-teleangiectasia and advanced stage high grade mature B-cell malignancies, Pediatr Blood Cancer 2014, 61: 360-362.

14. New HV, Cale CM, Tischkowitz M et al. Nijmegen breakage syndrome diagnosed as Fanconi anaemia, Pediatr Blood Cancer 2005, 44: 494-499.
15. Albert MH, Gennery AR, Greil J et al. Successful SCT for Nijmegen breakage syndrome. Bone Marrow Transplant 2010, 45: 622-626.

16. Al-Herz W, Bousfiha A, Casanova JL et al. Primary immunodeficiency diseases: an update on the classification from the International Union of Immunological Societies Expert Committee for Primary Immunodeficiency. Front. Immunol. 2014, 5: art.162.

17. Pfaff MW. Quantification strategies in real-time PCR. A - Z of quantitative PCR. 2004. Chapter 3: 87-112.

18. Swerdlow SH, Campo E, Harris NL et al. WHO Classification of Tumours of Haematopoietic and Lymphoid Tissues. IARC Pres, Lyon, France, 4th edition, 2008: 312-316.

19. Murphy SB. Classification, staging and end results of treatment in childhood non-Hodgkin's lymphoma: dissimilarities from lymphomas in adults. Semin in Oncology 1980, 7:332-339.

20. Schrappe M, Reiter A, Ludwig WD et al. Improved outcome in childhood acute lymphoblastic leukemia despite reduced use of anthracyclines and cranial radiotherapy: results of trial ALL-BFM 90. German-Austrian-Swiss ALLBFM Study Group. Blood 2000, 95: 3310-22.

21. Seidemann K, Tiemann M, Schrappe M et al. Short-pulse B-non-Hodgkin lymphoma-type chemotherapy is efficacious treatment for pediatric anaplastic large cell lymphoma: a report of the Berlin-Frankfurt-Munster Group Trial NHLBFM 90. Blood 2001, 97: 3699-3706.

22. Reiter A, Schrappe M, Tiemann M et al. Improved treatment results in childhood B-cell neoplasms with tailored intensification of therapy: a report of the Berlin-Frankfurt-Munster Group Trial NHL-BFM-90. Blood 1999, 94: 3294-3306.

23. Burkhardt B, Woessmann W, Zimmermann $\mathrm{M}$ et al. Impact of cranial radiotherapy on central nervous system prophylaxix in children and adolescents with central nervous system-negative stage III or IV lymphoblastic lymphoma. J Clin Oncol 2006, 24: 491-499.

24. Barovskaya Yu A, Aleinikova OV. Results of pediatric AML treatment according to the AML-MM 2006 in Belarus. Meditsinsky Journal, 2014, №4: 56-59. (In Russian)

25. Popov AM, Tsaur GA, Solodivnikov AG et al. Significance of different prognostic factors for occurrence of minimal residual disease in children with acute myeloblastic leukemia after induction therapy by the ALL-MB-2008 protocol. Voprosy Gematologii/Onkologii i Immunopatologii v Pediatrii, 2012; v.11: 20-27. (In Russian)

26. Conter V, Bartram CR, Valsecchi MC et al. Molecular response to treatment redefines all prognostic factors in children and adolescents with B-cell precursor acute lymphoblastic leukemia: results in 3184 patients of the AIEOP-BFM ALL 2000 study. Blood 2010, 115: 3206-14.

27. Gregorek H, Chrzanowska KH, Michalkiewicz J et al. Heterogeneity of humoral immune abnormalities in children with Nijmegen breakage syndrome: a 8-year follow-up study in a single centre. Clin Exp Immunol 2002, 130: 319-324. 
28. Gregorek H, Chrzanowska KH, Dzierzanowska-Fangrat $\mathrm{K}$ et al. Nijmegen breakage syndrome: long-term monitoring of viral and immunological biomarkers in peripheral blood before development of malignancy. Clin Immunol 2010, 135: 440-447.

29. Resnick I, Kondratenko I, Togoev O et al. Nijmegen breakage syndrome: clinical characteristics and mutation analysis in eight unrelated Russian families. J Pediatr 2002, 140: 355-361.

30. Kondratenko I, Paschenko O, Plyakov A et al. Nijmegen breakage syndrome. Adv Exp Med Biol 2007, 601: 61-67.

31. Kostyuchenko L, Makuch H, Kitsera N et al. Nijmegen breakage syndrome in Ukraine: diagnostics and follow-up. Centr Eur J Immunol 2009, 34: 46-52.

32. Distel L, Neubauer S, Varon R et al. Fatal toxicity following radio- and chemotherapy of medulloblastoma in a child with unrecognized Nijmegen breakage syndrome. Med Pediatr Oncol 2003, 41: 44-48.

33. Bakhshi S, Cerosaletti KM, Concannon P et al. Medulloblastoma with adverse reaction to radiation therapy in $\mathrm{Ni}$ - jmegen breakage syndrome. J Pediatr Hematol Oncol 2003, 25: 248-251.

34. Meyer S, Kingston H, Taylor A et al. Rhabdomyosarcoma in Nijmegen breakage syndrome: strong association with perianal primary site. Cancer Genet Cytogenet. 2004; 154: 169-174.

35. Chrzanowska KH, Digweed M, Sperling K et al. DNA-repair deficiency and cancer: Lessons from lymphoma. Hereditary tumors. From genes to clinical consequences. Edited by: Allgayer H., Rehder H., Fulda S. Weinheim: WILEY-VH; 2009: 377-391.

36. International Nijmegen breakage syndrome study group. Nijmegen breakage syndrome. Arch Dis Child 82: 400-406, 2000.

37. Gładkowska-Dura M, Dzierzanowska-Fangrat K, Dura WT et al. Unique morphological spectrum of lymphomas in Nijmegen breakage syndrome (NBS) patients with high frequency of consecutive lymphoma formation. J Pathol. 2008, 216: 337-44. 